\title{
A Contribution to the moth fauna (lepidoptera, heterocera) of Elazığ Province, Turkey
}

\author{
Erdem Seven a,* , Abdullah Çakır b \\ a Batman University, Department of Gastronomy and Culinary Arts, School of Tourism and Hotel Management, TR-72060, Batman Turkey \\ b Hilar Vocational and Technical Anatolian High School, TR-21950, Diyarbakır Turkey
}

\author{
Article history: \\ Keywords: \\ Lepidoptera \\ Moth \\ Maden \\ Elazı̆
}

ARTICLE INFO

Received 02 November 2018

Received in revised form 21 June 2019

Accepted 25 June 2019

\begin{abstract}
A B S TRACT
This study contributes to the Heterocera fauna of eastern Turkey. The paper presents a faunal list of 208 moth species belonging to 15 families, Arctiidae, Ctenuchidae, Cossidae, Drepanidae, Lasiocampidae, Lemoniidae, Lymantriidae, Notodontidae, Noctuidae, Psychidae, Pyralidae, Sphingidae, Thaumetopoeidae, Thyatiridae and Zygaenidae from Maden district of Elazı ğ Province. Among these moths, 150 species are recorded for the first time in Elazı $\breve{g}$ Province.
\end{abstract}

\section{Introduction}

The moth fauna of Turkey is represented by 5158 species in 67 families (Koçak \& Kemal, 2018). Information on the Turkish Lepidoptera fauna mostly based on the taxonomic and faunistic investigations. However, significant studies have been conducted in terms of finding out of the Turkish moth fauna. In this respect, examined and evaluated articles on the Heterocera fauna of Turkey and in point of the research region, are as follows: Staudinger $(1878,1881)$; Wagner $(1929,1930$, 1931); Pfeiffer (1931); Zukowsky (1941); Kansu (1963); Kornoşor (1982a, 1982b); Freina \& Hermann (1985); Hacker (1990); Koçak \& Seven (1994a, 1994b, 1996); Okyar \& Kornoşor (1994, 1997); Seven (1996); Kanat \& Kornoşor (2000) and Akkuzu et al. (2007).

The moth species of Elazığ Province have been poorly studied. Although, some sporadic agricultural studies have been carried out by Bolu \& Çınar (2005), Ayaz \& Yücel (2010) and Gegechkori \& Didmanidze (2015), first exhaustive research was published by Koçak and Kemal with the study "Synonymical and distributional list of the species of Elazığ Province" in 2007 and they were reported totally 199 moth species in their paper (Koçak \& Kemal, 2007). And following, they were reported 243 moth species in the latest current article "A synonymous and distributional list of the species of the Lepidoptera of Turkey" in 2018 (Koçak \& Kemal, 2018) from Elazığ Province. Seven \& Çakır (2018) also presented 8 Sphingidae species from the study area and 5 of them: Clarina kotschyi (Kollar, [1849]); Dolbina elegans A. Bang-Haas, [1913]; Laothoe populeti (Bienert, [1870]); Macroglossum stellatarum (Linnaeus, 1758) and Marumba quercus ([Denis \& Schiffermüller], 1775)) were added to the fauna of Elazığ.

In this paper, a total of 208 species of Heterocera species in the families, Arctiidae, Ctenuchidae, Cossidae, Drepanidae, Lasiocampidae, Lemoniidae, Lymantriidae, Notodontidae, Noctuidae, Psychidae, Pyralidae, Sphingidae, Thaumetopoeidae, Thyatiridae and Zygaenidae are listed faunistically from Maden district of Elazı̆g Province, eastern Turkey. And among them, 150 species are new reported for the fauna of Elazığ Province.

\section{Material and Method}

The materials in this study were collected between in AprilAugust 2016 and March-October 2017 at irregular intervals from Maden district of Elazığ Province with UV light traps. The species were diagnosed according to the literature data by considering the external morphological and genital structures of the materials. Samples are deposited in the special collection 
of the first author and in the Biology Laboratory of Batman University.

To identification and distribution of the species, the sources utilized are include: Wagner (1929, 1930, 1931); Kansu (1963); Freina \& Hermann (1985); Freina \& Witt (1987); Varga \& Ronkay (1991); Fibiger (1997); Ronkay et al. (2001); Hacker et al. (2002); Zilli et al. (2005); Leraut (2006, 2012); Fibiger \& Hacker (2007); Koçak \& Kemal (2007); Fibiger et al. (2009; 2010); Seven (2014); Kemal \& Koçak (2017); Koçak \& Kemal (2018); and Seven \& Çakır (2018).

\section{Results}

In this research, a total of 208 species are listed alphabetically under the related families. The new records for fauna of Elazığ Province are marked with " $\left({ }^{*}\right)$ " an asterisk.

\section{Faunal list of the species}

\section{Family Arctiidae}

1. *Arctia festiva (Hufnagel, 1766); 2. Arctia villica (Linnaeus, 1758); 3. *Manulea pseudocomplana (Daniel, 1939); 4. *Paidia rica (Freyer, [1855]); 5. *Phragmatobia fuliginosa (Linnaeus, 1758); 6. Phragmatobia placida (Frivaldsky, 1835).

\section{Family Ctenuchidae}

7. Dysauxes famula (Freyer, 1836).

\section{Family Cossidae \\ 8. *Dyspessa ulula (Borkhausen, 1790).}

\section{Family Drepanidae}

9. * Cilix asiatica A.Bang-Haas, 1907; 10. *Watsonalla binaria (Hufnagel, 1767).

\section{Family Lasiocampidae}

11. *Chondrostega palaestranum Staudinger, 1892; 12. Lasiocampa eversmanni (Kindermann, 1843); 13. *Lasiocampa grandis (Rogenhofer, 1891); 14. Malacosoma neustrium (Linnaeus, 1758); 15. *Phyllodesma tremulifolium (Hübner, [1810]).

\section{Family Lemoniidae \\ 16. Lemonia ballioni (Christoph, 1888).}

\section{Family Lymantriidae}

17. *Euproctis chrysorrhoea (Linnaeus, 1758); 18. *Euproctis melania (Staudinger, 1892); 19. *Leucoma wiltshirei Collenette, 1938; 20. Lymantria dispar (Linnaeus, 1758); 21. *Parocneria terebinthi (Freyer, [1838]); 22. Polymona lapidicola (Herrich-Schäffer, [1852]).

Family Notodontidae

23. * Clostera pigra (Hufnagel, 1766).

\section{Family Noctuidae}

24. Acantholipes regularis (Hübner, [1813]); 25. *Acontia titania (Esper, [1798]); 26. *Acontiola moldavicola (HerrichSchäffer, [1851]); 27. *Acronicta aceris (Linnaeus, 1758); 28. *Acronicta rumicis (Linnaeus, 1758); 29. *Aedophron phlebophora Lederer, 1858; 30. Aegle semicana (Esper, [1798]); 31. *Agrochola gratiosa (Staudinger, 1882); 32. *Agrochola pistacina (Goeze, 1781); 33. *Agrotis bigramma
(Esper, [1790]); 34. *Agrotis ipsilon (Hufnagel, 1766); 35. Agrotis obesa (Boisduval, 1829); 36. *Agrotis puta (Hübner, [1803]); 37. *Agrotis segetum ([Denis \& Schiffermüller], 1775); 38. *Allophyes asiatica (Staudinger, 1892); 39. *Ammoconia caecimacula (Fabricius, 1787); 40. *Amphipyra livida ([Denis \& Schiffermüller], 1775); 41. *Amphipyra micans Lederer, 1857; 42. *Anthracia eriopoda (Herrich-Schäffer, [1851]); 43. Apamea maraschi (Draudt, 1934); 44. *Apamea monoglypha (Hufnagel, 1766); 45. *Aporophyla australis (Boisduval, 1829); 46. *Atethmia centrago (Haworth, [1809]); 47. Autophila einsleri Amsel, 1935; 48. *Autophila ligaminosa (Eversmann, 1851); 49. *Behounekia freyeri (Frivaldsky, 1835); $\mathbf{5 0 .}$ *Calocucullia celsiae (Herrich-Schäffer, 1850); 51. * Calophasia barthae F.Wagner, 1929; 52. Calophasia platyptera (Esper, [1788]); 53. Caradrina bodenheimeri (Draudt, 1934); 54. *Caradrina clavipalpis (Scopoli, 1763); 55. *Caradrina flavirena (Guenée, 1852); 56. *Caradrina inumbrata (Staudinger, 1900); 57. *Caradrina kadenii (Freyer, 1836); 58. *Caradrina rjabovi (Boursin, 1936); 59. Catocala abacta Staudinger, 1900; 60. *Catocala elocata (Esper, [1787]); 61. *Catocala hymenaea ([Denis \& Schiffermüller], 1775); 62. *Catocala lupina HerrichSchäffer, [1851]; 63. *Catocala nymphagoga (Esper, [1787]); 64. Chazaria incarnata (Freyer, 1838); 65. *Cleonymia opposita (Lederer, 1870); 66. Clytie syriaca (Bugnion, 1837); 67. *Conistra ragusae (Failla-Tedaldi, 1890); 68. *Cosmia confinis Herrich-Schäffer, [1849]; 69. *Cryphia amasina (Draudt, 1931); 70. *Cryphia maeonis (Lederer, 1865); 71. *Cryphia occidentalis (Osthelder, 1933); 72. Cryphia ochsi (Boursin, 1941); 73. *Cryphia raptricula ([Denis \& Schiffermüller], 1775); 74. Cryphia receptricula (Hübner, [1803]); 75. *Diarsia rubi (Vieweg, 1790); 76. Dichagyris amoena (Staudinger, 1892); 77. *Dichagyris erubescens (Staudinger, 1892); 78. *Dichagyris nachadira (Brandt, 1941); 79. *Dichagyris nigrescens (Hofner, 1888); 80. *Dichagyris singularis (Staudinger, 1877); 81. Dichagyris squalidior (Staudinger, 1901); 82. *Dicycla oo (Linnaeus, 1758); 83. *Diloba caeruleocephala (Linnaeus, 1758); 84. Drasteria caucasica (Kolenati, 1846); 85. *Drasteria saisani (Staudinger, 1882); 86. *Drasteria sesquilina (Staudinger, 1888); 87. *Dryobotodes tenebrosa (Esper, [1789]); 88. *Dysgonia algira (Linnaeus, 1767); 89. *Dysgonia torrida (Guenée, 1852); 90. *Earias clorana (Linnaeus, 1761); 91. Egira anatolica (M.Hering,1933); 92. *Epilecta linogrisea ([Denis \& Schiffermüller], 1775); 93. *Episema didymogramma (Boursin, 1955); 94. Episema korsakovi (Christoph, 1885); 95. *Episema tersa ([Denis \& Schiffermüller], 1775); 96. *Eublemma aureola Fibiger \& Hacker, 2002; 97. Eublemma gratissima (Staudinger, 1892); 98. *Eublemma minutata (Fabricius, 1794); 99. *Eublemma ostrina (Hübner, [1808]); 100. Eublemma pallidula (HerrichSchäffer, 1856); 101. Eublemma panonica (Freyer, 1840); 102. *Eublemma parva (Hübner, [1808]); 103. Eublemma polygramma (Duponchel, [1842]); 104. Eublemma straminea (Staudinger, 1892); 105. Eublemma suppura (Staudinger, 1892); 106. Eugnorisma pontica (Staudinger, 1892); 107. *Eulocastra diaphora (Staudinger, 1879); 108. *Eutelia adoratrix (Staudinger, 1892); 109. *Euxoa cos (Hübner, [1824]); 110. *Euxoa nigrofusca (Esper, [1788]); 111. *Euxoa obelisca ([Denis \& Schiffermüller], 1775); 112. *Gortyna flavago ([Denis \& Schiffermüller], 1775); 113. *Hadena compta ([Denis \& Schiffermüller], 1775); 114. *Hadena gueneei (Staudinger, 1901); 115. *Hadena magnolii (Boisduval, 1828); 116. *Hadena ronkayorum Hacker, 1996; 117. Hadena roseocandida Hacker, 1996; 118. *Hadena sancta (Staudinger, 
1859); 119. *Hadena vulcanica (Turati, 1907); 120. Hadula mendica (Staudinger, [1895]); 121. *Haemerosia renalis (Hübner, [1813]); 122. *Hecatera spinaciae (Vieweg, 1790); 123. *Hecatera weissi (Boursin, 1952); 124. *Heliothis peltigera ([Denis \& Schiffermüller], 1775); 125. *Heterophysa dumetorum (Geyer, 1834); 126. *Hoplodrina ambigua ([Denis \& Schiffermüller], 1775); 127. *Hoplodrina octogenaria (Goeze, 1781); 128. *Hypena munitalis Mann, 1861; 129. *Leucania punctosa (Treitschke, 1825); 130. Leucochlaena muscosa (Staudinger, 1892); 131. *Lygephila amasina (Staudinger, 1879); 132. Lygephila craccae (Fabricius, 1787); 133. *Megalodes eximia (Freyer, 1845); 134. *Meganola gigantula (Staudinger, 1879); 135. *Meganola togatulalis (Hübner, 1796); 136. *Mesapamea didyma (Esper, [1788]); 137. *Mesogona acetosellae (Goeze, 1781); 138. *Mesoligia furuncula ([Denis \& Schiffermüller], 1775); 139. *Mesoligia literosa (Haworth, [1809]); 140. Metachrostis dardouini (Boisduval, 1840); 141. Metalopha gloriosa (Staudinger, 1892); 142. Mythimna alopecuri (Boisduval, 1840); 143. *Mythimna ferrago (Fabricius, 1787); 144. *Mythimna l-album (Linnaeus, 1767); 145. Mythimna vitellina (Hübner, [1808]); 146. *Noctua comes (Hübner, [1813]); 147. *Noctua janthina ([Denis \& Schiffermüller], 1775); 148. Noctua orbona (Hufnagel, 1766); 149. *Noctua pronuba (Linnaeus, 1758); 150. *Nola chlamitulalis (Hübner, [1813]); 151. *Olivenebula subsericata (Herrich-Schäffer, 1861); 152. *Oncocnemis confusa (Freyer, [1839]); 153. *Oncocnemis strioligera Lederer, 1853; 154. *Ophiusa lunaris (Goeze, 1781); 155. *Orthosia cerasi (Fabricius, 1775); 156. *Orthosia incerta (Hufnagel, 1766); 157. *Orthosia schmidtii (Diószeghy, 1935); 158. Ostheldera gracilis (Osthelder, 1933); 159. Pamparama acuta (Freyer, [1837]); 160. *Phylapora canescens (Duponchel, 1826); 161. *Plecoptera inquinata (Lederer, 1857); 162. *Polymixis serpentina (Treitschke, 1825); 163. *Polyphaenis propinqua Staudinger, [1896]; 164. *Polypogon proxima (Christoph, 1893); 165. Pseudenargia deleta (Osthelder, 1933); 166. *Pseudenargia regina (Staudinger, 1892); 167. Rhiza laciniosa (Christoph, 1887); 168. *Scotochrosta pulla ([Denis \& Schiffermüller], 1775); 169. *Setoctena dentula (Lederer, 1869); 170. *Shargacucullia verbasci (Linnaeus, 1758); 171. *Sideridis luteago ([Denis \& Schiffermüller], 1775); 172. *Simyra dentinosa Freyer, 1839; 173. *Spodoptera exiguum (Hübner, [1808]); 174. * Teinoptera oliva (Staudinger, [1895]); 175. *Tholera decimalis (Poda, 1761); 176. Thria robusta Walker, [1858]; 177. Tyta luctuosa ([Denis \& Schiffermüller], 1775); 178. *Valeria oleagina (Esper, [1786]); 179. *Valerietta boursini De Freina \& Hacker, 1985; 180. Victrix boursini (Draudt, 1936); 181. *Victrix duelduelica (Osthelder, 1932); 182. *Xanthia icteritia (Hufnagel, 1766); 183. *Xestia cohaesa (Herrich-Schäffer, [1849]); 184. *Xestia ditrapezium ([Denis \& Schiffermüller], 1775); 185. Xestia palaestinensis (Kalchberg, 1898); 186. *Xestia sareptana (Herrich-Schäffer, [1851]); 187. *Xestia xanthographa ([Denis \& Schiffermüller], 1775); 188. *Zekelita antiqualis (Hübner, [1809]); 189. *Zethes brandti Janzon, 1977; 190. *Zethes insularis Rambur, 1833; 191. *Zethes narghisa Brandt, 1938.

\section{Family Psychidae}

192. *Megalophanes viciella ([Denis \& Schiffermüller], 1775).

\section{Family Pyralidae}

193. Cynaeda gigantea (Staudinger, 1879); 194. *Nomophila noctuella ([Denis \& Schiffermüller], 1775); 195. *Pyralis farinalis (Linnaeus, 1758).

\section{Family Sphingidae}

196. Clarina kotschyi (Kollar, [1849]); 197. Dolbina elegans A.Bang-Haas, [1913]; 198. Laothoe populeti (Bienert, [1870]); 199. Macroglossum stellatarum (Linnaeus, 1758); 200. Marumba quercus ([Denis \& Schiffermüller], 1775); 201. Rethera komarovi (Christoph, 1885); 202. Smerinthus kindermanni Lederer, 1852; 203. Theretra alecto (Linnaeus, 1758).

Family Thaumetopoeidae

204. *Thaumetopoea solitaria (Freyer, [1838]).

\section{Family Thyatiridae}

205. *Polyploca korbi Rebel, 1901; 206. *Tethea ocularis (Linnaeus, 1767).

\section{Family Zygaenidae}

207. *Jordanita notata (Zeller, 1847); 208. *Zygaena graslini Lederer, 1855.

\section{Conclusions}

In this survey, Heterocera specimens collected from Maden district of Elazığ Province, eastern Anatolia of Turkey are evaluated, and 208 species are detected. Totally 150 species in the families, Arctiidae (4), Cossidae (1), Drepanidae (2), Lasiocampidae (3), Lymantriidae (4), Notodontidae (1), Noctuidae (126), Psychidae (1), Pyralidae (2), Thaumetopoeidae (1), Thyatiridae (2) and Zygaenidae (2) are recorded for the first time in Elazığ Province. The largest rate among the gathered samples includes Noctuidae species with $81 \%$. With these results, the moth fauna of Elazı $\breve{g}$ Province has reached to 393 species. When this number is compared to 5158 known moth species in the Turkish fauna (Koçak \& Kemal, 2018), it comprises approximately $9.6 \%$ of the total species. It is clearly seen that this study offers a serious contribution to the Lepidoptera of the region. But, when considering the vegetation and location of the field, it needs to be studied in more detail.

\section{References}

Akkuzu, E., Ayberk, H., and Inac, S., 2007. Hawk moths (Lepidoptera, Sphingidae) of Turkey and their zoogeogaphical distribution. J. env. biol., 28 (4), 723-730.

Ayaz, T., and Yücel, A., 2010. Elazığ İli Elma Alanlarında Bulunan Zararlı ve Yararlı Arthropod Türlerinin Belirlenmesi Üzerine Araştırmalar. Harran Üniversitesi Ziraat Fakültesi Dergisi, 14(1), 9-16.

Bolu, H., and Çınar, M., 2005. Elazığ, Diyarbakır ve Mardin İlleri Badem Ağaçlarında Zararlı Olan Lepidoptera Türleri, Doğal Düşmanları ve Önemlileri Üzerinde Gözlemler. Hr.Ü.Z.F.Dergisi, 9 (2), 63-67.

Fibiger, M., 1997. Noctuidae Europaeae. Vol. 3, Noctuinae. 418 pp., Entomological Press.

Fibiger, M., and Hacker, H., 2007. Noctuidae Europaeae. Vol. 9, Amphipyrinae, Condicinae, Eriopinae, Xyleninae. 410 pp., Entomological Press. 
Fibiger, M., Ronkay L., Steiner, A., and Zilli, A., 2009. Noctuidae Europaeae. Vol. 11, Pantheinae, Dilobinae, Acronictinae, Eustrotiinae, Nolinae, Bagisarinae, Acontiinae, Metoponiinae, Heliothinae and Bryophilinae. 504 pp., Entomological Press.

Fibiger, M., Ronkay L., Yela J.L., and Zilli, A., 2010. Noctuidae Europaeae. Vol. 12, Rivulinae, Boletobiinae, Hypenodinae, Araeopteroninae, Eublemminae, Herminiinae, Hypeninae, Phytometrinae, Euteliinae and Micronoctuidae. Inluding Supplement to Volumes 1-11. 451 pp., Entomological Press.

Freina, J.J. de, and Hermann, H., 1985. Neue Arten und Unterarten der FamilieNoctuidae ausAnatolien und Türkisch Kurdistan (Lepidoptera, Noctuidae). Entomofauna, 6 (19), 241-261.

Freina, J.J. de., and Witt, T., 1987. Die Bombyces und Sphinges der Westpalaerktis. Band 1: 47, 708 pp., Farbtafeln, 330 Karten; München.

Gegechkori, A. and Didmanidze, E., 2015. Has Ever Brahmaea Ledereri Rogenhofer, 1873 Inhabited the Colchis Refugium. American Journal of Environmental Protection. Special Issue: Applied Ecology: Problems, Innovations.Vol. 4, No. 3(1), 82-92.

Hacker, H., 1990. Die Noctuidae Vorderasiens. Beitrege Ökologie, Faunistik und Systematik von Lepidoptera. 707 pp., Apollo Books.

Hacker, H., Ronkay, L., and Hreblay, M., 2002. Noctuidae Europaeae. Vol. 4, Hadeninae I. 419 pp., Entomological Press.

Kanat, M., and Kornoşor, S., 2000. Kahramanmaras Noctuidae Faunası Üzerinde Sistematik Araştırmalar. Ç.Ü.Z.F. Dergisi, 15(2), 1-8.

Kansu, A.İ., 1963. Türkiye Lepidoptera faunası için ilkel liste IV. Bitki Koruma Bülteni, 3, 195-201.

Kemal, M., and Koçak, A.Ö., 2017. On the moths of Mutki district (Bitlis Province, East Turkey (Lepidoptera). Cesa News 150, 2-46.

Koçak, A.Ö. and Seven, S., 1994a. Türkiye Lepidoptera faunasına katkılar-I. Cent. ent. Stud., Misc. Pap., 20, 1-8.

Koçak, A.Ö. and S. Seven, 1994b. Türkiye Lepidoptera faunasına katkılar-II. Cent. ent. Stud., Misc. Pap., 21, 1-8.

Koçak, A.Ö. and Kemal, M., 2007. Synonymical and distributional List of the species of Elazığ Province (East Turkey) (Lepidoptera). Cent. ent. Stud., Misc. Pap. 113/114, 1-12.

Koçak A.Ö. and Kemal, M., 2018. A synonymous and distributional list of the species of the Lepidoptera of Turkey. Centre for Entomological Studies, Memoirs, 8, 1-487.

Kornoşor, S., 1982a. Türkiye Noctuidae (Lepidoptera) Türleri ve Yayılışlarına Ait Ön Liste, I. Trifidae Grubu. Ç.Ü. Fen-Edebiyt Fak. Yıllığı, Sayı, 1-2, 74-95.

Kornoşor, S., 1982b. Türkiye Noctuidae (Lepidoptera) Türleri ve Yayılıșlarına Ait Ön Liste, II. Quadrifidae Grubu. C..Ü. Fen-Edebiyt Fak. Yıllığı, Sayı, 1-2, 95-102.
Leraut, P., 2006. Moths of Europe, volume 1, Saturnids, Lasiocampids, Hawkmoths, Tiger Moths ... 387 pp., N. A. P. Editions.

Leraut, P., 2012. Moths of Europe. Volume 3 Zygaenids, Pyralids 1. 599pp. 201 text-figs., 112 pls. N.A.P. ed., Verrières-le-Buisson.

Okyar, Z., and Kornoşor, S., 1994. Trakya Bölgesi Noctuidae (Lepidoptera) türlerinin tespiti çalıșmaları I. XII. Ulusal Biyoloji Kongresi 6-8 Temmuz 1994/Edirne, 217-224.

Okyar, Z. and Kornoşor, S., 1997. Trakya Bölgesi Noctuidae (Lepidoptera) familyası türlerinin tespiti çalışmaları II. Türk. entomol. derg., 21(3), 197-212.

Pfeiffer, E., 1931. Einleitung. [in] Osthelder,L. \& E.Pfeiffer, Lepidopteren-Fauna von Marasch in türkisch Nordsyrien. Mitt. münch. ent. Ges. 21 (2), 68-81.

Ronkay, L., Yela, j.L., and Hreblay, M., 2001. Noctuidae Europaeae. Vol. 5, Hadeninae II. 452pp., Entomological Press.

Seven, E., 2014. Siirt'in Şirvan ilçesinin Macroheterocera türleri üzerine eko-faunistik araştırmalar (Lepidoptera). Doktora tezi. $428 \mathrm{~s}$. Yüzüncü Yll Univ. Fen Bil. Enst., Van [unpublished].

Seven, E., and Çakır, A., 2018. A Faunistic Study On the Hawk Moths (Sphingidae, Lepidoptera) Of Maden District (Elaziğ Province, East Turkey). 1st International Engineering and Technology Symposium (1st Iets), 03-05 May, p. 209.

Seven, S., 1996. Türkiye Lepidoptera faunası ve ekolojisi Üzerine araştırmalar-I. Cent. ent. Stud., Priamus, 8 (1/2), 1-52.

Staudinger, O., 1878. Lepidopteren-Fauna Kleinasien's. Horea societatis Entomologicae Rossicae, 14, 176-482.

Staudinger, 0., 1881. Lepidopteren-Fauna Kleinasien's. Horea societatis Entomologicae Rossicae, 16, 65-135.

Varga, Z., and Ronkay, L., 1991. Taxonomical notes on the genus Victrix Staudinger, 1879 (Lepidoptera, Noctuidae). II. The subgenus Rasihia Koçak, 1989. Nota lepid., 14 (2), 144-170.

Wagner, V.F., 1929. Weiterer Beitrag zur Lepidopteren-Fauna InnerAnatoliens. Mitteilungen der Münchner Entomologischen Gesellschaft, 1, 57-80.

Wagner, V.F., 1930. Zweiter (III) Beitrag zur Lepidopteren-Fauna Inner-Anatoliens. Int. Ent. Zeit., 24, 545-558.

Wagner, V.F., 1931. Dritter (IV) Beitrag zur Lepidopteren-Fauna InnerAnatoliens. Int. Ent Zeit., 24, 467-493.

Zilli, A., Ronkay, L., and Fibiger, M., 2005. Noctuidae Europaeae: Apemeini. Vol. 8, 323 pp., Entomological Press.

Zukowsky, B., 1941. Sivas und Akşehir 1937 (Lep). Zeitschrift des wiener Entomologenvereins, 267-272. 